\title{
Finding Centre of Gravity of Yoga Asana Postures to support Self-Assisted Yoga Practice
}

\author{
K. Ponmozhi , P. Deepalakshmi , V. Vallinayagi
}

\begin{abstract}
Self-assisted yoga practice should ensure safety. Being in a wrong posture while doing practice may cause problems like fall or fractures in the bones. Practitioners of activities like yoga, sports etc. should maintain correct postures all through their practice. Maintaining a correct posture is influenced by Centre of Gravity whose location is based on distributing the entire body weight evenly for stability. In this paper, we propose a method to find the Centre of Gravity of any asana sequence. The location of Centre of Gravity will be used by the assistive system to instruct the practitioner how to alter their postures to avoid fall and enable to take-up perfect practice. Motion capture systems will give the details in the form of Biovision hierarchy format, from which the coordinate values of human joints can be accessed. Based on the segmentation method, whole body Centre of Gravity is calculated. We have used Vrikshasana for our experiment and yoga posture data for ten participants are used to find the Centre of Gravity variations. Data for two trails were acquired. CM Variations in segments like thigh, angle are calculated. From the results the instructors may find whether ankle or thigh's position should be changed to maintain correct posture.

Keywords : Assisted Living, Bio- Mechanics, Bio Vision Hierarchy, Centre of Gravity, Segmented Method, Yoga.
\end{abstract}

\section{INTRODUCTION}

G on members of their family. This dependency to do every activity on others makes them home ridden. Assistive technology comes in handy for these people to alleviate this dependency to some extent. This paper adds corrective mechanism based on Centre of Gravity for self-assisted yoga practice. The Centre of Gravity location in other words the location based on which the entire body weight should be distributed for stability.

All living and non-living objects are affected by universal gravitation. Mechanical interactions with the environment and within the biological system influence the motion and function of all the objects in the universe. Biomechanics is

Revised Manuscript Received on December 05, 2019

* Correspondence Author

K. Ponmozhi*, Department of Computer Applications Kalasalingam Academy of Research and Education Krishnankoil,Srivilliputhur,India chezhiyan71.p@gmail.com

P. Deepalakshmi, Department of Computer Science and Engineering, School of Computing, Kalasalingam Academy of Research and Education Krishnankoil, Srivilliputhur, India deep.kumar@klu.ac.in

V. Vallinayaki, Assistant Professor, PG and Research Department of Computer Science, Sri Sarada college for Women,Tirunelveli, Tamil Nadu, India. the study related to the study of influence of mechanics on the body's movement, shape at all levels ranging from molecular level to system level [1].

Posture of a human body can be defined by the joints position. Coordinated interactions of the various parts of musculoskeletal system such as bones, muscles, and joints are essential to achieve human body movement [2]. As human posture changes, Centre of gravity will also change based on current posture. Balance is based on the position of centre of gravity of the human body. Factors that are contributing to the stability are in general, (i) the size of base of support, (ii) the horizontal distance from the edge of the support of the Centre of Gravity (COG), (iii) height of COG, (iv) weight of the body, (v) direction of the base in relation to the force.

Yoga has been considered as a means to strengthen, align and balance the structure of body, also enhance the stabilization of the body during activities. Some of the yoga asana have been used as remedial activity for issues like lower-back pain [3]. Yoga improves hip and spinal flexibility which helps in reducing lowering back pain [4-5].In many a times, yoga has been practiced by individuals without trainers' assistance. When people do self-assisted yoga asana practice, there may be a possibility of fall because of miss balancing; this may be due to wrong position of Centre of Gravity. And thus, it is useful and essential to understand and know the correct place of Centre of Gravity for every asana posture and this will be helpful for preventing injury, correcting abnormality, healing and rehabilitation.

In this paper, segmental method of centre of gravity calculation is used as this method is flexible and useful in calculating centre of gravity even in the complex actions. Also, we can use segmental method when the subject is under external forces.

The centre of gravity of Trigonansana postures are calculated for 10 participants with 5 male and 5 female of age ranging from 20 to 50 . Their average weight is $58.3 \mathrm{~kg}$, average height is $160 \mathrm{~cm}$, and average age is 35.2 . Some participants at the age greater than 45 are not able to maintain this posture. To make this posture, some of the participant bent their body in the lateral directions. This is because to maintain their centre of gravity along the leg, some weight has been shifted in the lateral direction. 
In this paper, section II discusses bio-mechanics and directional representations and section III describes the segments of human body and calculation of centre of gravity based on the segments. Section IV discusses the results and in section $\mathrm{V}$, we provided conclusion about our findings.

\section{BACKGROUND}

\section{A. Selecting Representation of yoga postures}

As yoga asana are based on sequence of postures performed on a time series, it is convenient to represent them in any one of the motion representation formats. The format used in this paper is BVH (Bio-Vision Hierarchy), which represents the human skeleton as hierarchy of joints. Any BVH file has two parts namely (i) initial posture's of the asana (ii) motion part which have the transformation matrices. From that format, the coordinate values of every joint can be accessed. It is convenient to calculate the centre of mass of every segment as discussed in section III with these coordinate values for the particular posture. Fig. 1 gives the sample representation of a human being at anatomical position, where we consider hips as root and chest as well as neck joints as its children. Every joint will have offset values of their position from their parent joint. Values given after the keyword OFFSET are displacement values along $\mathrm{x}, \mathrm{y}, \mathrm{z}$ axis respectively. From these values the joint coordinate values can be calculated using following coding.

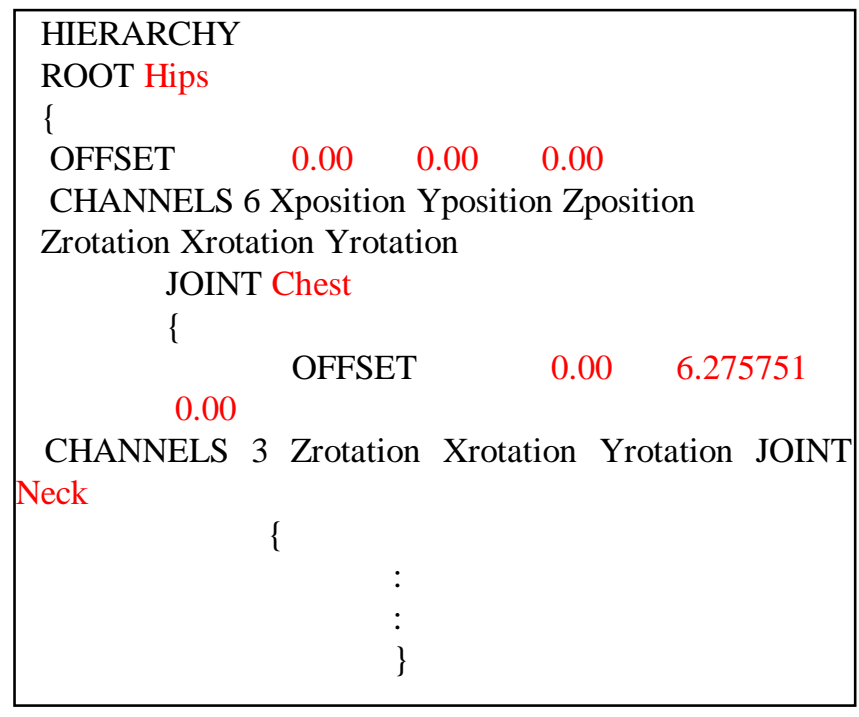

In motion part, the values of angle values for rotation, translation vector values will be given for every joint, and for every frame. Based on these values, it is possible to do transformations on the previous frames, and find the posture of the current frame. There is a possibility to mention the frame rate while capturing the motion, and thus the number of frames. If the same posture has to be maintained for a specific amount of time, that can also be represented in frame time.

\section{B. Bio-mechanics of Human Body}

Centre of gravity is a point around which human body weight is evenly distributed. This is an imaginary point and does not belong to any part of the body. Sometimes this point may lay outside the body based on the posture. The force of gravity on human body is downward through our body's centre of gravity. In anatomical position, centre of gravity lies below the waist i.e. in lower torso as shown in Fig 1.Generally, Centre of gravity location depends upon the weight, height and distribution of the weight in the position concerned.

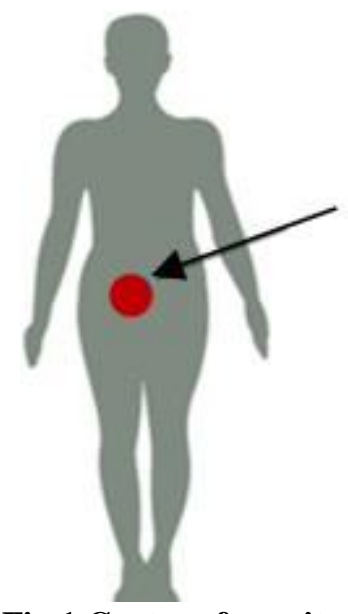

\section{Centre of Gravity}

Fig.1 Center of gravity location in anatomical position

The area beneath the human body which touches the earth or the supporting surface is termed as base support. Usually the base support will be any one of these: (i) both the feets, (ii) one feet, (iii) both arms, (iv) single arm, (v) stomach or (vi) in combination of these. Figure 2, shows the base support parts.
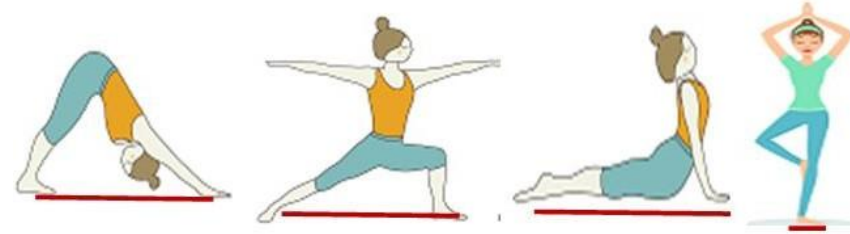

Fig 2: Base support points (a) feet and hands (b) both feet (c) lower body and hand (d) one foot

Mid-point of these base support points is called as centre of support. Whenever line of gravity goes outside the Centre of Support (COS), human body will not be in a balanced/stable position.

Centre of gravity is proportional to weight of the body. Usually this centre of gravity will be closer to the side that carries most weight. For example, though we stand in both feet support, if we put all our weight in one leg then the centre of support is that leg and centre of gravity passes through that leg.

Common facts about centre of gravity are: 1. Larger the base support, there is a possibility of centre of gravity falling inside the base line, 2.Center of gravity moves to the side where there is heavy load or weight 3 . If the height of centre of gravity is lower and near to the base support, it is more stable than compared to higher centre of gravity.

There is a correlation between stride width and fall [13]. While doing asana, the placement of body weight or otherwise the centre of support has be in alignment with the centre of gravity. If not, the practitioner may fall. Thus, it is essential to know the location of centre of gravity while doing yoga and also other physical exercises. 


\section{RELATED WORK}

Body Segmental parameters that influence the centre of gravity are (i) segmental length, (ii) segmental weight. By using these parameters, it is possible to find the centre of gravity of any posture in any complicated motion sequence such as applicable to yoga asana too. Human segmental parameters can be obtained using mathematical methods [14] or using data of MRI [15] to find parameters of 14 body segments.

Trajectory of centre of gravity may also be estimated using a human body modeled as four-link object [16]. Identifying the centre of gravity position while the body is in motion is essential for the areas such as space motion, rehabilitation, finding ways to improve work efficiency.

Reference data of percentage distribution of segment lengths to human total height has been measured by Zatsiorsky and de Leva [18] by using segmentation methods. Data collection method used by them may not be suitable for complicated postures and for all body types. Methods mentioned in the above papers are using high-technology equipments. So it may not be suitable for all conditions.

In this paper, we are trying to use mathematical calculations to estimate complicated posture sequences available in the form of motion captured data. These data will have coordinate values of every joint. By applying the formulae, we can calculate the centre of gravity.

\section{PROPOSED SYSTEM}

\section{A. Finding Centre of Gravity of a posture}

Factors that are contributing to the stability are in general, (i) the size of base support, (ii) the horizontal distance from the edge of the support of the centre of gravity (COG), (iii) height of centre of gravity, (iv) weight of the body, (v) direction of the base in relation to the force.

Location of centre of gravity changes based on the posture of human body $[8,9]$.It combines the mass and centre of gravity location of every segment of the body. Human body has been represented as collection of connected rigid segments $[10,11]$. Every segment will have varying weight based on the posture. Every segment's length can be represented in terms of percentage of whole body height

\section{B. Segment length}

Over all body mass distribution is considered as a function of mass distribution within every segment of a particular posture. To find mass distribution of a segment, it is essential to find the location of centre of the mass of every segment.

The distance of body parts such as shoulder, elbow, wrist, hand etc. from the centre of the body can be represented by proximal and distal. Proximal is the point or joint of any segment which is the one closer to (i) midline or another reference point (may be base support, or hand, shoulder etc.). Distal is the point or joint which is further away from (i) midline or (ii) another reference point. For example, with reference to the (midline) centre of our body - the torso, the walking, running, and exercise or training sequences. This is

attachment point shoulder is proximal than elbow, whereas hand is distal from torso compared to wrist, elbow and shoulder.

Using Table.1, every segment's proximal and distal joints can be used to find the Centre of Mass of that segment. Because of symmetric nature of human body, upper arm values are applicable to both right and left upper arm. The same applies to forearm, hand, thigh, lower leg and foot.

Formula for calculating segment centre of mass is given in Eqn. (1) and Eqn. (2).

$$
\begin{aligned}
& \text { XCOG } \\
& (\text { degmenti } \\
& \left(\text { distal } l_{\text {gegmenti }}-\text { Xproximal }_{\text {gegmenti }}\right) * \\
& (\% \mathrm{~cm} \text { of segmenti })+\text { Xproximal }
\end{aligned}
$$

\section{YCOGsegmenti $=(Y$ distal_segment $i-$ Yproximal_segmenti $) *(\% \mathrm{~cm}$ of segmenti) + Yproximal_segmenti (2)}

By applying the values of this centre of mass of every segment in Eqn. (3) and (4), one can find centre of mass of the whole body.

$$
\text { XCOMwholebody }=\frac{\sum_{\mathrm{i}} \text { Mi } \bullet \text { XCoGsegmenti }}{\Sigma_{\mathrm{i}} \text { Mi }}
$$

YCOMwholebody $=\frac{\sum_{i} \text { MixYCOGi }}{\sum_{i} M i}$

\begin{tabular}{|c|c|c|c|}
\hline \multirow[t]{2}{*}{ Segment } & \multicolumn{2}{|c|}{ Center of Gravity (\%Length) } & \multirow[t]{2}{*}{ Joints } \\
\hline & Males & Females & \\
\hline Head \& Neck & 50.02 & 48.41 & Head and neck \\
\hline Trunk & 43.1 & 37.82 & $\begin{array}{l}\text { Center of } \\
\text { shoulder joints } \\
- \\
\text { Center of Hip } \\
\text { joints }\end{array}$ \\
\hline Upper Arm(s) & 57.72 & 57.54 & $\begin{array}{l}\text { Shoulder joint - } \\
\text { Elbow joint }\end{array}$ \\
\hline Fore $\operatorname{Arm}(\mathrm{s})$ & 5.74 & 5.59 & $\begin{array}{l}\text { Elbow joint - } \\
\text { Wrist joint }\end{array}$ \\
\hline Hand & 8 & 74.74 & $\begin{array}{l}\text { Wrist Joint - } \\
\text { Finger Tip }\end{array}$ \\
\hline Thigh & 40.95 & 36.12 & $\begin{array}{l}\text { Hip joint - knee } \\
\text { joint }\end{array}$ \\
\hline Shank & 3.95 & 43.52 & $\begin{array}{l}\text { Knee joint - } \\
\text { Ankle joint }\end{array}$ \\
\hline Foot & 4.15 & $\overline{40.14}$ & Toe $^{\text {Ankle - }}$ \\
\hline
\end{tabular}

Where i ranges from 1 to 14 (from head to foot, all body segments will be considered). The segments and their proximal and distal end points are given in table 1 .

Assume that the Proximal and Distal coordinate values of thigh segment is represented as $\mathrm{Xw}, \mathrm{Yw}$ (waist) and $\mathrm{Xk}, \mathrm{YK}$ (Knee). \%centimeter is the ratio value of the centimetre of particular segment in the total height can be accessed from table (1). From Equ.(4) and (5), the centre of mass for the segment thigh can be calculated using Equ. (5) and Equ. (6). 


$$
\begin{aligned}
& \text { XCOGthigh }=(\text { Xknee }- \text { Xwaist }) *(\% \mathrm{~cm})+\text { Xwaist } \\
& \text { YCOGthigh }=(\text { Yknee }- \text { Ywaist }) *(\% \mathrm{~cm})+\text { Yknee }
\end{aligned}
$$

\section{Centre of Gravity in YOGA posture}

Centre of support is the midpoint of base support. In normal standing posture, it will be in the centre point between our feet. If the centre of gravity and centre of base support of the human body lies in one point or if both are aligned vertically in same line, then balance is assured. While practicing every yoga postures, one has to maintain his/her centre of support and centre of gravity be aligned on the same line.

Two or more motor mechanisms contribute to maintaining human body balance. Central nervous system of human is responsible for balancing human body, which gives feedback actions to control the change in centre of gravity of human body. Usually these feedback actions will be given to hip, knee and ankle joints. Their movements will counteract the changes in centre of gravity [6].

Counter actions or postural changes because of sudden external disturbances can be of two categories namely (i) ankle strategy, which is in antero-posterior (A/P) direction, and changes the ankle but little variations in hip or knee and (ii) hip strategy, in which changes will be in hip position than in ankle and knee, also it is in the mediolateral (M/L sometimes in antero-posterior direction) [7]. In general, changes in mediolateral direction is controlled by hip joint and antero-posterior direction is controlled by ankle joints.

Information related to the centre of gravity and the amount of movement required by every joint in order to maintain balance is essential, to correct the misaligned postures. For example in case of trigonasana, practitioner has to lift one leg from the floor and place it on the knee of the other leg. It will be useful if the trainer knows whether adjustments to hip joints, knee joints or ankle joints are needed during this movement.

To maintain this balance one may spread the weight from one weight bearing point to the other. The fact is that lower centre of gravity height will ensure balance. And thus whenever possible, it is advisable to bring the centre of gravity closer to the ground. In case of tree pose, one has to bring the centre of gravity back to the standing foot.

According the standard tree pose described in[3], the participant lift one foot off the ground, eventually working their foot up to their calves, and then above their knees. The pressure of the foot exerted on the inner thigh causes the body reaction force to swing in the $\mathrm{M} / \mathrm{L}$ direction. In such case, the body slightly moves to the side of the standing leg.

Yoga poses rejuvenate every human body part. The posture we considered here is Vrikshasana, also known as the Tree Pose. While doing this asana practitioner will stretches the legs, arms, and the back which help them to get better balance [19].

For the posture shown in Fig.3, we marked the joint points, and extracted the coordinate values for those proximal and distal joint points of every segment is provided in the Table. 2 .Using formulae $[1,2,3,4]$ we can find the

centre of Gravity of the whole body. In the reverse inference, we can find the joint values changes in the hip, knee, ankle joints so that whether Mediolateral or antero-posterior directional move is required to maintain balance.

Image will be normalized to 500X500 pixel sizes, the points extracted from the image are shown in fig.3.

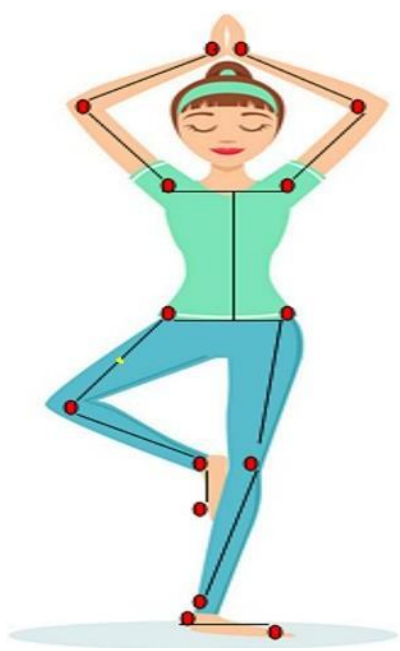

Fig.3: Vrikshasana

Table II. Coordinate Values of the Joints for the Example Figure

\begin{tabular}{|l|l|l|}
\hline 1. & Left wrist, & 231,31 \\
\hline 2. & Right wrist & 259,31 \\
\hline 3. & Left elbow & 126,76 \\
\hline 4. & Right elbow & 357,76 \\
\hline 5. & Left shoulder & 192,76 \\
\hline 6. & Right shoulder & 293,138 \\
\hline 7. & Left hip & 203,243 \\
\hline 8. & Right hip & 198,241 (proximal) \\
\hline 9. & Left knee & 293,138 \\
\hline 10. & Right knee & 112,308 (distal) \\
\hline 11. & Left ankle & 224,465 \\
\hline 12. & Right ankle & 222.355 \\
\hline 13. & Left heel & 216,479 \\
\hline 14. & Right heel & 240,354 \\
\hline 15. & Left toe & 290,487 \\
\hline 16. & Right toe & 234,397 \\
\hline 17. & Right finger tip & 246,4 \\
\hline 18. & Left finger tip & 241,4 \\
\hline 19. & Base of neck & 248,124 \\
\hline 20. & Head top & 244,58 \\
\hline
\end{tabular}

Segment's mass is given as percentage of total mass of the body which is represented by Mi.Similarly a segment's length is given as percentage length of the total height.

$\mathrm{X}$ value of Centre of gravity of Segment Thigh = Xhip $($ proimal $)+($ length $\%) *($ Xknee $($ distal $)-$ proximal $)$

As per the measurement of Fig.3 given in Table.2,

$\mathrm{X}$ value of Centre of gravity of Segment Thigh $=198+$ $(112-198) * .433=160.762$ 
$\mathrm{Y}$ value of Centre of gravity of Segment Thigh = $\mathrm{Y}_{\text {hip }}$ (proximal) + (length $\left.\%\right) *\left(\mathrm{Y}_{\text {knee }}(\right.$ distal $)-$ $Y_{\text {hip }}($ proximal))

$\mathrm{Y}$ value of center of gravity of segment thigh $=241+$ $(112-198) * .433=224.87$

Similarly, every segment's centre of gravity can be found for a particular posture. From BVH file, we can find the coordinate values of every proximal and distal joint values of every segment. The motion part of the BVH file will give the values of transformations to be applied on the previous frame coordinate values. And thus, it is possible to find the centre of gravity of every posture of yoga.

\section{IMPLEMENTATION}

The BVH file for Trigonasan has been passed as input to the function, centre of gravity Calculator. Anatomical position is the first portion of the file. Motion part of the file will be processed and coordinate values of each segment's distal and proximal values can be calculated.

Input: BVH files of trigonansana

Output: Array -Segment_COM [1..14 ]

,FrameCOG[1..30]

1. Extract coordinate information of every segment from input BVH file

2. Store the distal and proximal end values of every segment in the variable

3. Calculate COG of the segment using the formula given in Equ.1 and Equ. 2

4. Store it in array Segment_COM, iterate steps 2 and 3 for all segments

5. Find the COG of the posture using Equ.3 and Equ.4, and store it in array FrameCOG

6. Iterate steps from 1 to 5 for all the frames

In the BVH file, hip has been considered as centre and its coordinate value is $(0,0,0)$. For the purpose of calculation, we have treated the foot at $(0,0,0)$. All the coordinates are changed accordingly. The distal and proximal values of every segment will be stored in the array. Values of 14 segments, as mentioned earlier, are stored in the array. By applying Equ.1 and Equ.2 individual segments centre of gravity are calculated and stored. Finally, the centre of gravity location of the posture will be calculated using this array. Similarly for every frame, the cog will be calculated.

\section{RESULTS}

Participants height and cog measurement during Anatomical position is shown in Fig. 4, the height of $\operatorname{cog}$ as percentage of total body height is shown in Fig.5. Based on this centre of gravity for 10 participants in Vrikshasana are calculated .

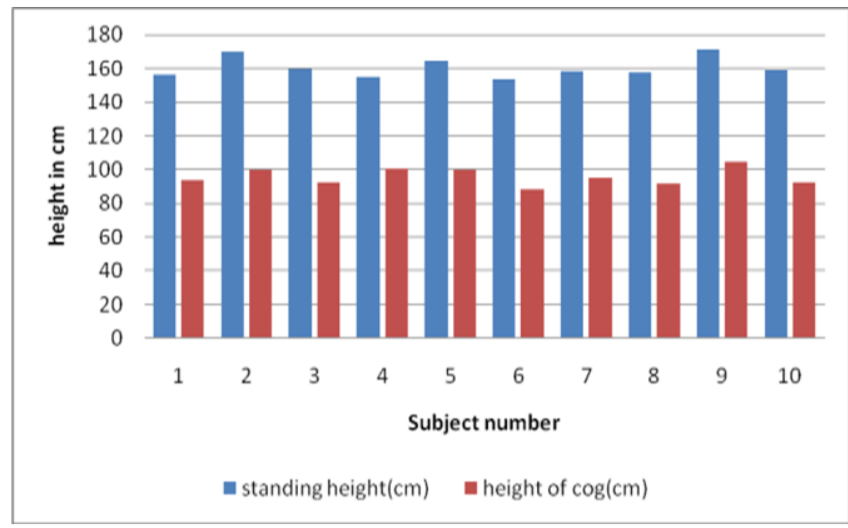

Fig.4: Height and cog height at anatomical position

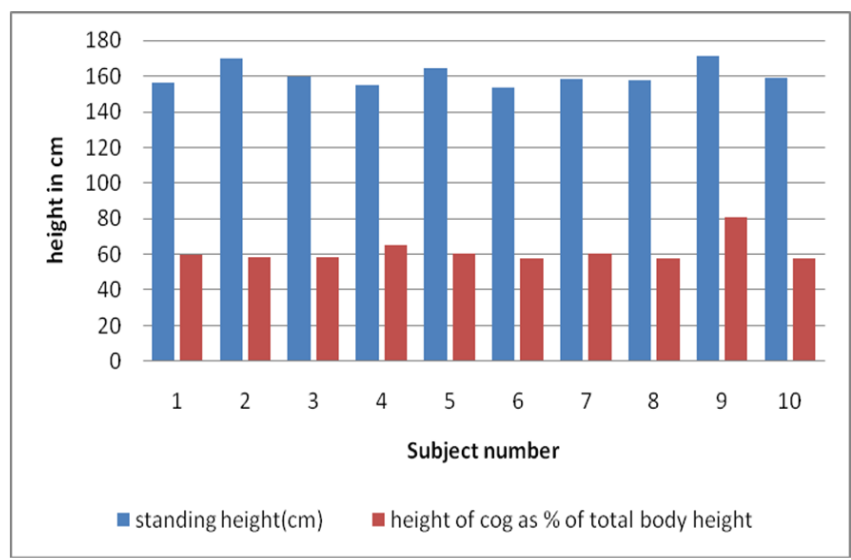

Fig 5: height and cog height in percentage of total height (at anatomical position)

In Vrikshasana, depending upon the leg which is footed on the floor, the foot will have different value than the other leg. This is because in the leg which is placed near the knee of the other, the distance between proximal and distal value is lesser compared to the footed leg.

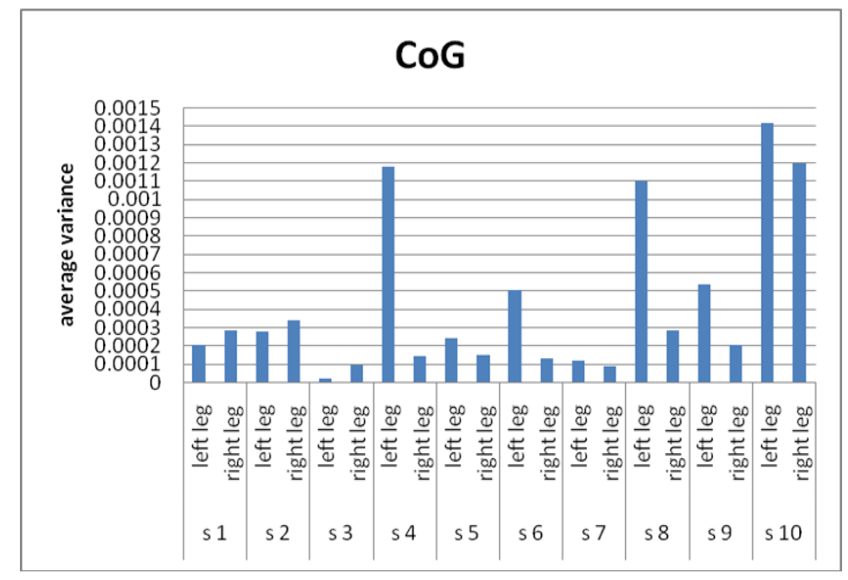

Fig. 6: Average variance of $\operatorname{cog}$ of 2 trails

As seen from Fig.6, the correlations between cogs on the two trails shows that subject 3 maintains the cog at the same location regardless of the leg in which the asana was performed 


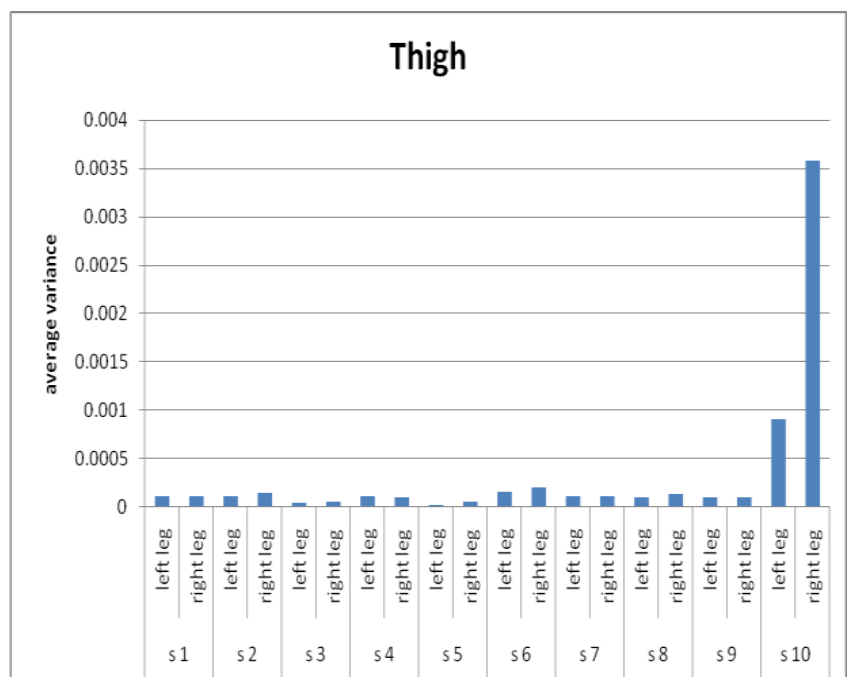

Fig.7: Average variance of $\operatorname{cog}$ in segment thigh of 2 trails

From Fig.7, we can infer that subject 3 and 5 balanced both thighs properly, in both the case regardless of the leg which is used for doing asana. In the case of subject 10 , it is convenient for subject 10 while doing asana in left leg than in right leg. They find it difficult to balance, they could not place the leg near to the knee of the standing leg, and this leads to very large variance.

\section{CONCLUSION}

For human body stability, it is important to know the centre of gravity location. Every postural change will have its effect on centre of gravity. In the case of yoga asana, it is essential to know the change of cog location in order to maintain balance. It is also essential to prevent fall or fracture too. In this paper, we proposed a method to find $\operatorname{cog}$ for Vrikshasana postures. This method can be used for all the asana. 10 participants with two trails were made. Based on the motion captured data for these trails, the average variance of cog locations of ankle, thigh and the whole body cog location of the participants were calculated and shown. In future, the effect and the weight bearing points for the positional changes during asana postures will be calculated so that the practitioners may be guided to shift their weight to those weight bearing points in order to avoid fall.

\section{REFERENCES}

1. Tung-Wu Lu, Chu-Fen Chang. Biomechanics of human movement and its clinical applications. Kaohsiung Journal of Medical Sciences (2012) 28, S13-S25.

2. Watkins J. Structure and function of the musculoskeletal system. Champaign: Human Kinetics; 1999

3. Cramer H, Lauche R, Haller H, Dobos G. A systematic review and meta-analysis of yoga for low back pain. Clin J Pain. 2013;29:450-60.

4. Williams K, Abildso C, Steinberg L, Doyle E, Epstein B, Smith D, et al. Evaluation of the effectiveness and efficacy of Iyengar yoga therapy on chronic low back pain. Spine (Phila Pa 1976) 2009;34:2066-76.

5. Galantino ML, Bzdewka TM, Eissler-Russo JL, Holbrook ML, Mogck EP, Geigle P, et al. The impact of modified Hatha yoga on chronic low back pain: A pilot study. AlternTher Health Med. 2004;10:56-9.

6. Nashner LM, McCollum G. The organization of human postural movements: A formal basis and experimental synthesis. Behav Brain Sci $1985 ; 8: 135-72$.

7. Gatev P, Thomas S, Kepple T, Hallett M. Feedforward ankle strategy of balance during quiet stance in adults. J Physiol 1999;514:915-28
8. D. Lafond, M. Duarte, F. Prince - "Comparison of three methods to estimate the center of mass during balance assessment". Journal of Biomechanics, 37, 9,September 2004, 1421-1426.

9. Shimba T-"An estimation of center of gravity fromforce platform data", 2010.10.Journal of Biomechanics, 17, 1984, 53-60.

10. Hanavan, E. P. (1964);"A mathematical model of the human body". AMRL-TR Aerospace Medical Research Laboratories, WrightPatterson Air Force Base, Ohio.

11. Miller, D.I. and Morrison, W. (1975);"segmental parameters using the Hanavan human body model". Med. Sci. Sports4. Stoudt H,

12. Damon A, McFarland, and Roberts

13. Clauser, C. E., McConville, J. T., and Young, J.M.;"Weight, volume, and center of mass of segmentsof the human body",(1969)

14. Krebs DE,GoldvasserD,LockertJD,PortneyLG,Gill-Body, Is base of support greater in unsteady gait?, Phys Ther. 2002 Feb;82(2):138-47

15. D.I.Miller, A Comouter simulation model of airborne phase of diving, PH.D. dissertation. Pennsylvania State University, 1970.

16. W.H Ho, C.C.,Lee and T,Y. Shiang, "The study of segment of human body of Taiwanese Young People from MRI". Journal of Medicial and Biological Engineering, 24(S): s1-s6, 2004.

17. D.Lenzi, A.Cappello and L.Chiari,. "Influence of body segment parameters modeling assumption on the estimate of center of mass trajectory", Journal of Biomechanics, 36:1335-1341, 2003.

18. Paolo de Leva (1996) Adjustments to Zatsiorsky-Seluyanov's Segment Inertia Parameters. Journal of Biomechanics 29 (9), pp. 1223-1230.

19. Zatsiorsky, V. M., Seluyanov, V. N. and Chugunova, L. G.: Methods of de-termining mass-inertial characteristics of human body segments, Contemporary Prob-lems of Biomechanics, (Edited by Chemyi G. G. and Regirer, S. A.), CRC Press,Massachusetts, 272\{291, 1990.

20. Allhoff F, Swan LS, editors. Yoga - Philosophy for Everyone: Bending Mind and Body. Sussex, UK:Wiley; 2012.

\section{AUTHORS PROFILE}

Dr. K. Ponmozhi is currently working as Assistant professor in the Department of computer applications, Kalasalingam Academy of Research and Education, Srivilliputhur, TamilNadu, India. She received her Bachelors degree in computer science from Madurai Kamaraj University, her MCA from Bharathidasan University. She received her Ph.D in computer science from Mother Teresa women's University. She has more than 20 years of teaching experiences. She published many articles in journals and conferences both in National level and International Level. Her area of interest is networking, computer graphics, image processing, cognitive science.

Dr.P.Deepalakshmi is currently working as a Professor in Department of Computer Science and Engineering at Kalasalingam Academy of Research and Education (KARE), Virudhunagar, Tamilnadu, India. She is also serving as Dean, School of Computing. Her research interest includes Optimization Techniques, Network Routing, Distributed Computing, Network Security, Data Analytics, Machine Learning Techniques. She also takes care of KARE ACM student chapter as faculty mentor. Contact her at deepa.kumar@klu.ac.in

Dr. V. Vallinayagi was Graduated from Bharathidasan University in 1989 and completed M.Sc (Computer Science) in 1991 under the same University. She received M.phil and Ph.D respectively in Computer Science in the year 2004 and 2017 respectively from Manonmanium Sundaranar University,Tirunelveli-11 India. She is having more than two decades of teaching experience and 5 years of research experience. She has been invited as a speaker for many workshops, acted as a chairperson for many conferences. She is guiding $8 \mathrm{Ph} . \mathrm{D}$ scholars and 3 M.Phil Scholars. I have Guided and produced 13 M.Phil Scholars. She published 35 Research papers which includes national, International Journals and conferences. She has authored three books. She is one of the member in Board of studies of many educational institutions. Currently She is working as an Assistant Professor, PG and Research Department of Computer Science, Sri Sarada college for Women, Tirunelveli-11.She is Serving as Head and Vice principal of Sri Sarada college. 